\title{
Bestimmung des Brechungsindex des Schmiermittels Beruforge 152DL unter Verwendung eines dafür entwickel- ten Dünnschichtnormals
}

\author{
Sebastian Metzner ${ }^{1}$, Tamara Reuter ${ }^{1}$, Elisa Wirthmann ${ }^{1}$, Tino Hausotte ${ }^{1}$ \\ ${ }^{1}$ Lehrstuhl für Fertigungsmesstechnik, Friedrich-Alexander-Universität Erlangen-Nürnberg, \\ Nägelsbachstraße 25, 91052 Erlangen, Deutschland
}

\begin{abstract}
Zusammenfassung
Für eine Messung von blechmassivumgeformten Bauteilen möglichst nahe am Fertigungsprozess spielen systematische Abweichungen durch den darauf noch verbliebenen Schmierstoff eine signifikante Rolle. Die erwartete Schichtdicke dieses Schmierstoffs liegt bei den betrachteten Bauteilen in einem Bereich von unter $35 \mu \mathrm{m}$. Für die Bestimmung des Brechungsindex des Schmiermittels und somit die Auswirkungen des Schmierstoffs auf die interferometrische Messung, wird ein Dünnschichtnormal vorgestellt, welches in einem Bereich von 6 bis $100 \mu \mathrm{m}$ einen kontinuierlichen Messbereich abbildet. Mit dem eingesetzten Messverfahren, einer koaxialen interferometrischen Schichtdickenmessung, können so in verschiedenen Abständen die optische Dicke bestimmt werden. Daraus lässt sich im Anschluss der Brechungsindex näherungsweise bestimmen. Zudem können die Abweichungen der angezeigten Schichtdicke bei bekanntem Brechungsindex nun bewertet werden.
\end{abstract}

Keywords: Brechungsindex, Interferometrie, Schmiermittel Beruforge 152DL, Dünnschichtstufennormal

\section{Einleitung}

Die Blechmassivumformung (BMU) als neuartiges Fertigungsverfahren stellt die optische Messtechnik vor Herausforderungen [1]. Vor allem bei Messungen möglichst nahe am Fertigungsprozess beeinflussen Faktoren wie das verwendete Schmiermittel das Messergebnis. Typischerweise wird bei der BMU das Schmiermittel Beruforge 152DL (BF152DL) verwendet. Da die Auswirkungen des Schmiermittels auf optische Messsysteme meist die Kenntnis des Brechungsindex voraussetzen, ist dessen Bestimmung für weitere Untersuchungen notwendig.

\section{Aktueller Forschungsstand zur Messung dünner Schichten}

Bisher werden hauptsächlich Messsysteme, welche auf Ellipsometrie oder Reflektometrie basieren, für die Schichtdickenmessung und somit auch für eine mögliche Brechungsindexbestimmung eingesetzt. Die Grundlagen hierfür werden von McCrackin [2] und Hlubina [3] beschrieben. Aufgrund der langen Messdauer dieser Methoden werden jedoch zunehmend interferometrische Messsysteme verwendet [4]. Grundlage für die Bestimmung des Brechungs- index ist die geometrische Schichtdickenmessung. Bei der interferometrischen Schichtdickenmessungen wird zunächst zwischen Einschicht- und Mehrschichtsystemen unterschieden. Bei Mehrschichtsystemen erfolgt die Trennung der Signale der einzelnen Schichten meist mittels Fourier Analyse unter Anwendung des Algorithmus der Fast Fourier Transformation (FFT). Quinten [5] beschrieb die Grenzen der FFT bei Schichtdickenmessungen durch Aliasund Leakage-Effekte. Diese Einflüsse können durch eine Korrektur reduziert aber nicht vollständig vermieden werden. Ghim und Kim [6] zeigten eine verbesserte Version der dispersiven Weißlichtinterferometrie, die es ermöglicht, den Brechungsindex ohne Kenntnis der geometrischen Dicke zu messen. Die Schichtdicke wird dabei aus den spektral aufgelösten Interferenz-Signalen gewonnen. Cardin und Leduc [7] nutzten zur Brechungsindexbestimmung unter anderem die Prismen-FilmKoppler-Spektroskopie, welche auf der Verwendung von Transfermatrizen basiert und gleichzeitig die Bestimmung des Brechungsindex, der Schichtdicke und die optischen Verluste der gemessenen Schicht zulässt. Die von Metzner in [8] vorgestellten Themen zur Auswirkung von dünnen Schmierstoffschichten auf Streifenlichtprojektionssysteme zeigen eine Abweichung der Messergebnisse in einem betrachteten Bereich von 
$10 \mu \mathrm{m}$ bis $100 \mu \mathrm{m}$. Ab einer Schichtdicke von $40 \mu \mathrm{m}$ konvergiert die gemessene systematische Abweichung gegen diesen Wert, da die Strahlen der Streifenlichtprojektion im inneren der Schicht teilweise diffus reflektiert oder absorbiert werden.

In der vorliegenden Arbeit werden neben der Bestimmung des Brechungsindex und der Abweichungen zur geometrischen Schichtdicke auch die Grenzen des Systems bei dem eingesetzten Schmierstoff aufgezeigt.

\section{Verwendetes Messsystem und entwickeltes Dünnschichtnormal}

Für die eigentliche Dünnschichtmessung wird das Prinzip der koaxialen interferometrischen Schichtdickenmessung verwendet. Der verwendete Sensor CHRocodile 2S der Firma Precitec erreicht bei einem Messfleckdurchmesser von $13 \mu \mathrm{m}$ eine vertikale Auflösung von $10 \mathrm{~nm}$ und ist für einen Messbereich von $3 \mu \mathrm{m}$ bis $180 \mu \mathrm{m}$ optischer Dicke ausgelegt. Die Strahlen treffen bei diesem Messprinzip lotrecht auf die transparente Schicht und werden dort teilweise reflektiert, teilweise durchdringen sie die Schicht und werden an der darunterliegenden Fläche reflektiert. Durch das polychromatische Licht variiert die Phasenverschiebung zwischen den reflektierten Teilstrahlen mit der Wellenlänge, wodurch konstruktive und destruktive Interferenz entsteht. Wird die Intensität über die Wellenzahl aufgetragen entsteht eine harmonische Funktion. Die Frequenz dieser Funktion ist proportional zur optischen Weglänge der Schicht. Durch die bereits beschriebene Anwendung der Fourier Analyse ist es möglich, bei mehreren Schichten die Funktionen zu trennen und die Frequenzen zu bestimmen. Daraus kann anschließend bei bekannter Brechzahl die einzelnen Schichtdicken bei einem Mehrschichtsystem errechnet werden [9].
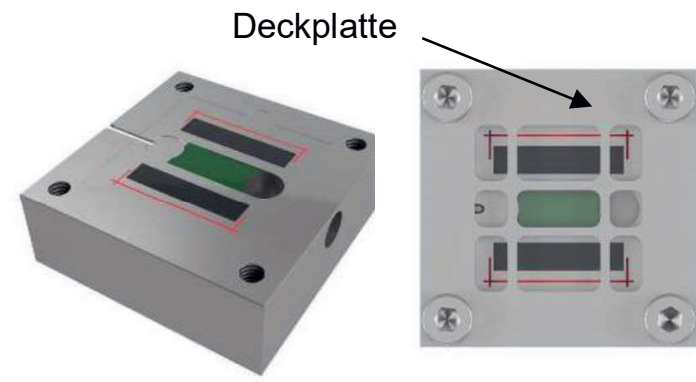

Abb. 1: Dünnschichtnormal als CAD-Modell mit den farblich markierten Funktionsflächen

Zur Abbildung der erwarteten Schichtdicken in einem definierten Bereich wurde angelehnt an DIN 32567 und DIN 5436 für die Messung von
Dünnschichten ein Dünnschichtnormal entwickelt. Das Dünnschichtnormal, welches in Abbildung 1 dargestellt ist, wurde aus Edelstahl mit einer Abmessung von $30 \mathrm{~mm} \times 30 \mathrm{~mm} \times$ $10 \mathrm{~mm}$ realisiert. Zur Messung der Schichtdicken wurde in der Mitte des Modells eine Keilform gefräst (grün). Der Keil deckt einen Dickenbereich von $6 \mu \mathrm{m}$ bis $100 \mu \mathrm{m}$ ab. Die absoluten Schichtdicken werden im Vergleich zu den beiden Bezugsebenen (schwarz) bestimmt, die neben dem Keil angeordnet sind. Die Zuleitung des Schmierstoffes erfolgt über ein Loch an der Stirnseite des Keils, in das ein kurzer Zuleitungsschlauch mit Verschluss angebracht wird. Referenzlinien (rot) gewährleisten eine reproduzierbare Messung auf den verschiedenen Positionen des Keils.

In Abbildung 2 ist das reale Dünnschichtnormal, das mit dem Schmierstoff BF152DL gefüllt ist, dargestellt. Eine Deckplatte drückt eine dünne Glasscheibe auf das Modell, wodurch ein Austreten des Schmierstoffs an den Seiten des Keils verhindert wird.

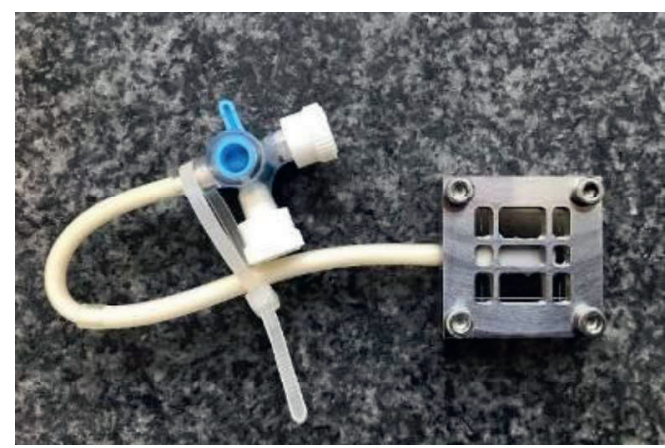

Abb. 2: Mit Schmierstoff gefülltes Dünnschichtnormal mit montierter Glasund Deckplatte sowie Zuflussschlauch.

Durch die Verstrebungen und der Verwendung einer $100 \mu \mathrm{m}$ dicken Deckplatte wird außerdem ein Aufwölben der Deckschicht verhindert. Vergleichsmessungen mit einer dünnen Folie zeigen eine deutliche Aufwölbung und dadurch eine Verfälschung der Messergebnisse.

\section{Untersuchter Schmierstoff Beruforge 152DL}

Der untersuchte Schmierstoff ist ein Einschichtschmierstoffsystem welcher aus anorganischen und organischen Komponenten besteht. Der Schmierstoff der auf Basis von Wasser ist, ist mit Wachspartikeln versehen, um die Reibung während der Umformung positiv zu beeinflussen. Bei ausreichendem Druck platzen diese Partikel auf und verringern dabei die Reibung während des Umformprozesses. Vor allem bei großen Prozesskräften und unterschiedlichen 
Umformgraden wie es bei der BMU der Fall ist, ist eine druckabhängige Steuerung der tribologischen Eigenschaften sinnvoll. Nach der Umformung wird der Schmierstoff wieder zähflüssiger und führt bei der optischen Messung zu systematischen Messabweichungen in Form der Schichtdicke des Schmierstoffs. Die erwarteten Schichtdicken des Schmierstoffs liegen in der hier vorgestellten Anwendung der BMU in einem Bereich von $1 \mu \mathrm{m}$ bis $35 \mu \mathrm{m}$.

\section{Vorgehen zur Bestimmung des Brechungs- index und eingesetzter Messaufbau}

Die Bestimmung des Brechungsindex erfolgt in Anlehnung an DIN 32567-5. Die geometrische Dicke $d_{g e o}$ ergibt sich dabei aus der Division aus der geometrischen Dicke $d_{\text {opt }}$. und dem Brechungsindex $n$ :

$$
d_{g e o}=\frac{d_{o p t}}{n}
$$

Bei bekannter optischer und geometrischer Dicke kann der Brechungsindex berechnet werden. Die Bestimmung der optischen Schichtdicke mit dem wellenlängenabhängigen Brechungsindex wird dabei mit folgender Formel realisiert:

$$
d_{o p t}=\frac{m}{2 \cdot\left(v_{\max }-v_{\min }\right)}
$$

mit minimaler und maximaler Wellenzahl $v_{\text {min }} /$ $v_{\text {max }}$ und Pixelnummer der Spektralanalyse $m$. Die Wellenzahl ist als Inverse zur Wellenlänge definiert. Um die geometrische Dicke an dem entwickelten Dünnschichtnormal zu bestimmen, wird das Stufennormal zunächst mit dem kalibrierten Fokus-Variationsmesssystem Alicona InfiniteFocus gemessen. Abbildung 3 zeigt das geometrische Modell. Die Referenzlinien an den Seitenflächen des Modells sind dabei gut zu erkennen. Dadurch ist ein wiederholtes Messen der Schichtdicke an verschiedenen Positionen des Keils möglich.

Die möglichen Schichtdicken auf dem Keil liegen in einem Bereich von $6 \mu \mathrm{m}$ bis $100 \mu \mathrm{m}$. Die geometrische Dicke des Deckglases wird mit $100 \mu \mathrm{m}$ angegeben.

Die Versuche werden auf der am Lehrstuhl vorhandenen Nanopositionier- und Nanomessmaschine SIOS NMM-1 durchgeführt. Mithilfe dieses Systems kann der Sensor mit einer Positionierunsicherheit von wenigen Nanometern zu dem Modell positioniert werden. Zudem ist eine Synchronisierung der Maschine mit dem Messsignal möglich, wodurch eine Abtastung der Oberfläche im Positionierbereich denkbar ist.

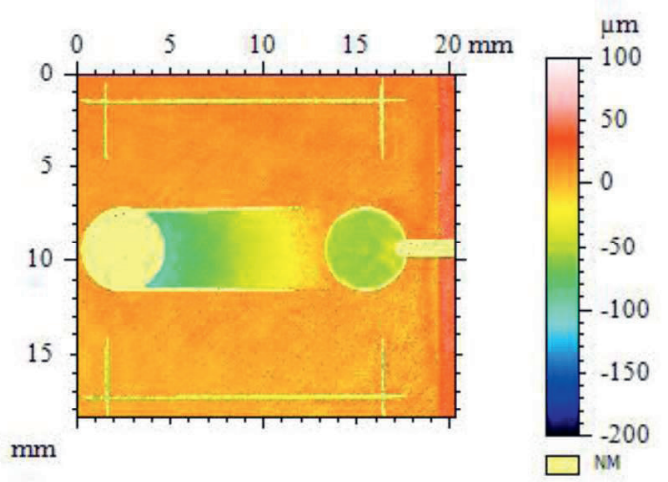

Abb. 3: Oberflächenmodell des Stufennormals gemessen mit dem Alicona InfiniteFocus

Abbildung 4 zeigt den Messaufbau auf der Nanomessmaschine mit dem Dünnschichtnormal. Durch die Referenzmarker ist eine wiederholbare Positionierung des Messsystems auf den verschiedenen Keilpositionen möglich.

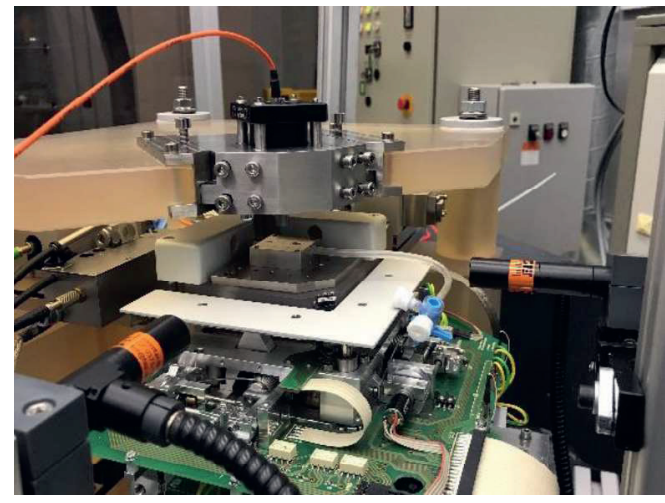

Abb. 4: Nanomessmaschine NMM-1 mit integrierten interferometrischen Messkopf und Stufennormal

\section{Ergebnisse der Brechungsindexbestim- mung bei der Dünnschichtmessung}

Da der Brechungsindex noch unbekannt ist, werden zunächst Wiederholmessungen zur Bestimmung der optischen Dicke durchgeführt. Bei der Beurteilung des Messgerätes werden Messungen mit Luft und mit Wasser als Referenzschichten mit jeweils 5 Wiederholungen in einem Bereich von $6 \mu \mathrm{m}$ bis $30 \mu \mathrm{m}$ durchgeführt. Abbildung 6 zeigt die Abweichung der Schichtdicken in Bezug zur geometrischen Dicke an. Der Brechungsindex wird dabei mit 1,33 bei Wasser und mit 1,00 bei Luft angenommen. Die Standardabweichung ist dabei bei Wasser, unabhängig von der Schichtdicke größer als bei der Messung der Luftschicht. 


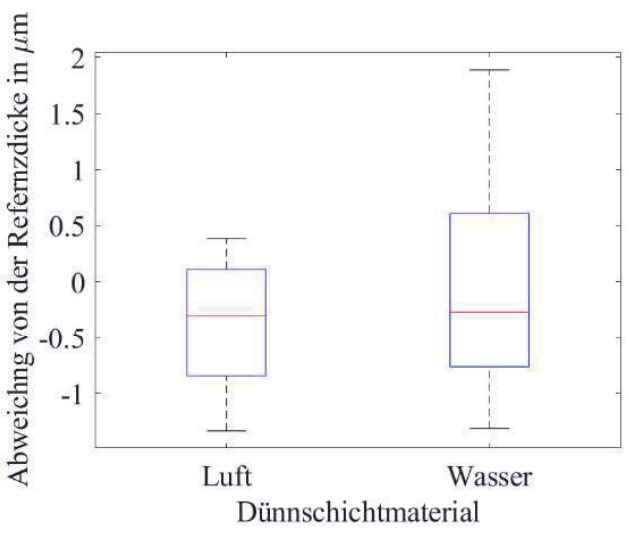

Abb. 6: Abweichungen zur absoluten Schichtdicke bei Luft und Wasser als Medium

Gründe für die erhöhte Standardabweichung ist die Wellenlängenabhängigkeit des Brechungsindex. Dieser wird aufgrund der Anwendung der FFT als konstant angenommen, ist aber abhängig von der Wellenlänge. Dies kann zu Abweichungen in der Bestimmung der Schichtdicke führen.

Die Bestimmung der optischen Schichtdicke mit dem wellenlängenabhängigen Brechungsindex würde mit folgender Formel erfolgen:

$$
d_{f f t}=\frac{m}{2 \cdot\left(\frac{n\left(\lambda_{\min }\right)}{\left(\lambda_{\min }\right)}-\frac{n\left(\lambda_{\max }\right)}{\left(\lambda_{\max }\right)}\right)}
$$

mit $\lambda_{\min } / \lambda_{\max }$ als minimale und maximale Wellenlänge des Weißlichtspektrums. Durch das verwendete Weißlichtinterferometer ist jedoch eine Berechnung des wellenlängenabhängigen Brechungsindex nicht möglich.

Bei intransparenten Schmierstoffen ist zusätzlich der Effekt der Absorption zu beachten. Wird von einem absorbierenden Material ausgegangen, so wird der komplexe Brechungsindex $N$ mit dem Extinktionskoeffizienten $k$ berechnet [10]:

$$
N=n+j k
$$

Die insgesamt sehr geringen Abweichungen zeigen jedoch unabhängig von der Wellenlängenabhängigkeit des Brechungsindex die Fähigkeit des Messsystems in dem definierten Messabstand die genauen Höhenwerte zu ermitteln.

Abbildung 7 zeigt den nach (1) berechneten Brechungsindex für Luft, Wasser und dem Schmierstoff BF152DL in Abhängigkeit der absoluten Schichtdicke.

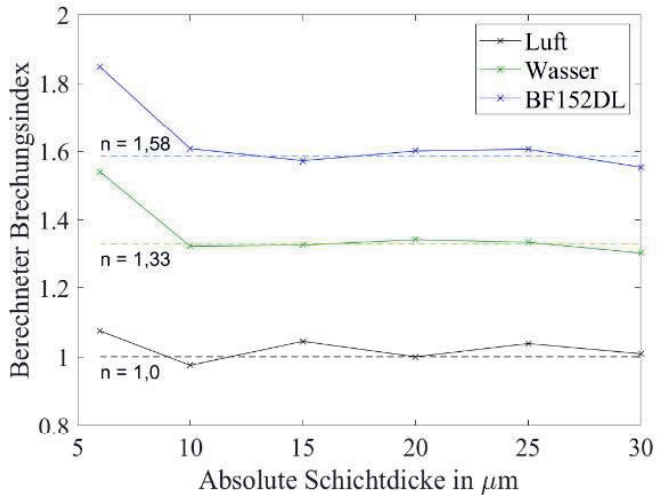

Abb. 7: Abhängigkeit des berechneten Brechungsindex in Abhängigkeit der gemessenen Schichtdicke

Dabei fällt die systematisch erhöhte Abweichung bei einer absoluten Schichtdicke von $6 \mu \mathrm{m}$ auf. Eine Bestimmung des Brechungsindex kann nach DIN 32567-5 bei kleinen Schichtdicken zu einer Abweichung des Brechungsindex vom wahren Wert führen. Die Ergebnisse der hier aufgezeigten Messungen bestätigen diese These. Die Brechzahl des Schmiermittels konvergiert erst ab einer Schichtdicke von $10 \mu \mathrm{m}$ gegen die erwartete Brechzahl. Der Brechungsindex des Schmierstoffes wird daher mit 1,585 $\pm 0,079$ bestimmt.

\section{Ergebnisse bei der Messung der absoluten Schichtdicke des Schmierstoffes}

Nachdem der Brechungsindex bestimmt wurde, ist eine Messung des gesamten Dickenbereichs des Keils möglich. Dazu werden Wiederholmessungen mit den drei Schichtarten Luft, Wasser und Schmierstoff durchgeführt. Abbildung 8 zeigt die Abweichungen der gemessenen und berechnetet Schichtdicken von der absoluten Schichtdicke.

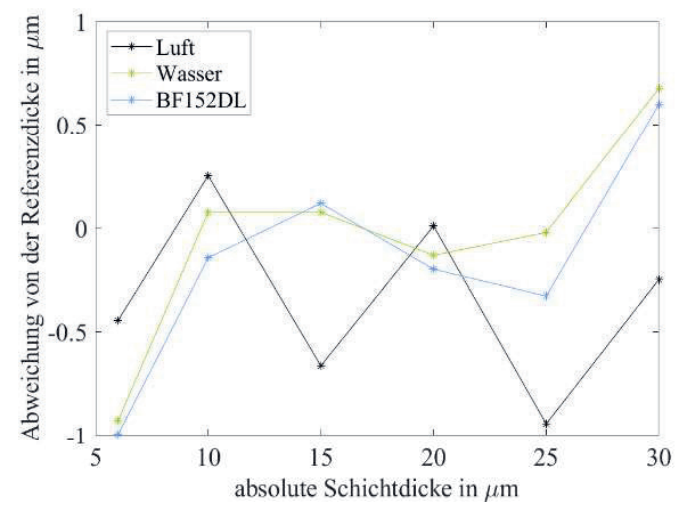

Abb. 8: Abweichungen zur absoluten Schichtdicke bei Luft, Wasser und BF152DL als Medium 
In der Abbildung ist eine leicht negative Abweichung zur absoluten Schichtdicke zu erkennen. Die Schichtdicken Wasser und BF152DL haben dabei einen ähnlichen Verlauf mit einer großen Abweichung bei einer Schichtdicke von $6 \mu \mathrm{m}$. Diese Abweichung entspricht bei einem Brechungsindex $>0$ aber der erwartenden Abweichung bei dünnen Schichten. Abbildung 9 stellt die Standardabweichungen bei den einzelnen Widerholmessungen im gesamten Dickenbereich des Keils dar.

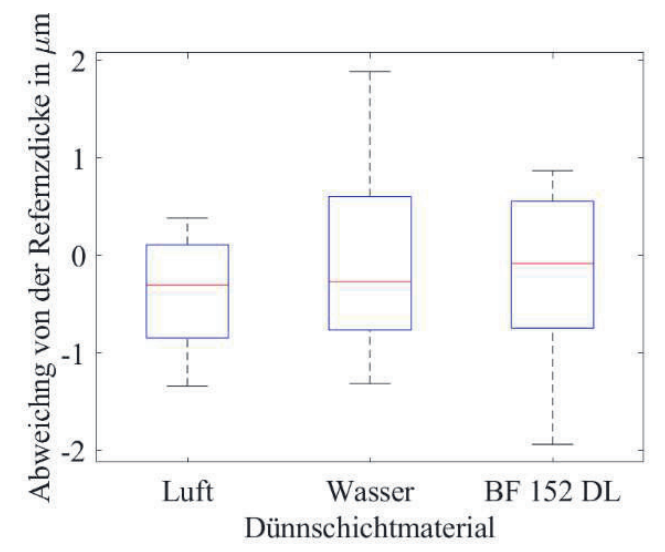

Abb. 9: Abweichungen zur absoluten Schichtdicke bei Luft und Wasser als Medium

Dabei sind die Standardabweichungen bei Wasser und BF152DL im Vergleich zur Messung der Schichtdicke mit Luft erhöht. Die erhöhten Streuwerte sind durch die unterschiedlichen Reflexionen der Lichtstrahlen an der Grenzfläche zwischen den Schichten zu erklären. Grundsätzlich weisen die Ergebnisse die erwartete Abhängigkeit der Schichtdicke von der geometrischen Höhe des Keils mit einer mittleren Abweichung von unter $1 \mu \mathrm{m}$ auf. Die Standardabweichungen der Schichtart Luft liegen unter 0,5 $\mu \mathrm{m}$. Im Vergleich dazu liegen die Standardabweichungen der Schichtart Wasser und BF152DL bei ca. 1,0 $\mu \mathrm{m}$.

Die erhöhten Standardabweichungen sind zum einen durch ein stärkeres Umgebungsrauschen zu begründen. Zudem ist eine eindeutige Detektion der Peaks im Leistungsspektrum bei zunehmender Schmierstoffschichtdicke oft nicht möglich. Abbildung 10 zeigt beispielhaft ein aufgenommenes Leistungsspektrum des Schmierstoffs bei einer geometrischen Schichtdicke von $10 \mu \mathrm{m}$. Hierbei ist eine Bestimmung der Schichtdicke lediglich durch die Differenz der Schichtdicke der Deckschicht von der Gesamtschichtdicke möglich.

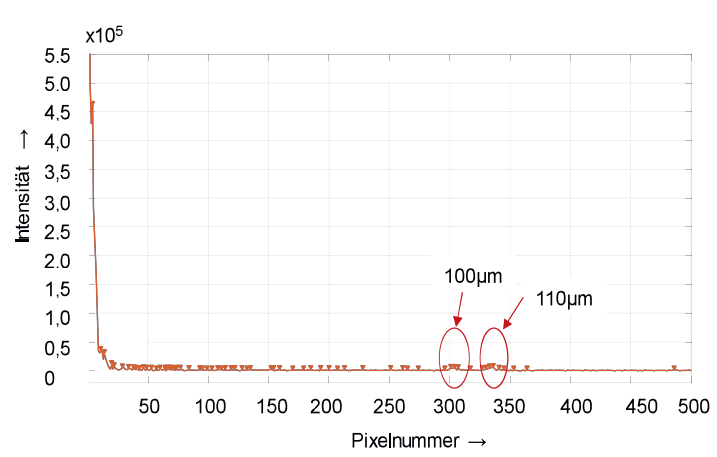

Abb. 10: Leistungsspektrum der Schmierstoffs BF152DL bei einer geometrischen Schichtdicke von $10 \mu \mathrm{m}$

Erst bei Betrachtung von lokalen Maxima des Leistungsspektrums (Abbildung 11) ist ein Peak der Schichtdicke erkennbar.

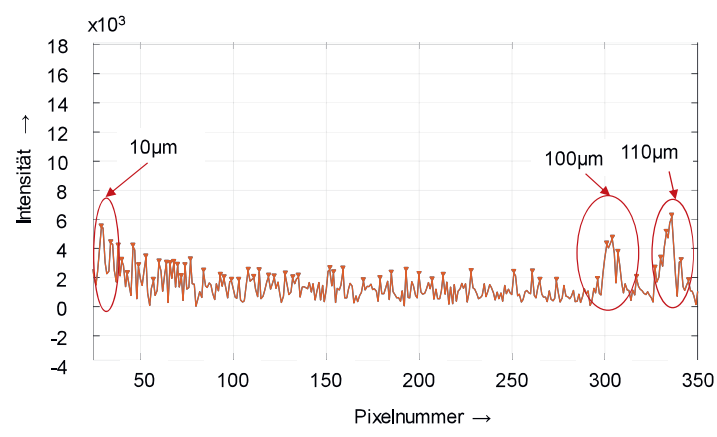

Abb. 11: Lokale Maxima des Leistungsspektrums zeigen keine eindeutige Ausprägung von Peaks

Dies ist mit der Absorption der Strahlung in der Schmierstoffschicht zu begründen. Durch die erhöhte Absorption des Signals ist das SignalRauschverhältnis deutlich schlechter, was zu einer verminderten maximal messbaren Schichtdicke führt. Zudem werden an den Wachspartikeln in dem Schmierstoff die einfallenden Strahlen diffus reflektiert. Dies führt dazu, dass die reflektierten Strahlen nicht mehr vom Detektor aufgenommen werden, und, dass das Erkennen der Peaks schlechter wird

\section{Danksagung}

Die Arbeit wird durch die Deutsche Forschungsgemeinschaft (DFG) im Rahmen des Sonderforschungsbereichs SFB/TR73 in dem Teilprojekten A6 gefördert und wurde an der Friedrich-Alexander-Universität Erlangen-Nürnberg erstellt. 


\section{Literaturnachweis}

[1] Metzner S., Ramesh R., Müller A., Hausotte T.: Evaluation of reflection properties of sheet bulk metal formed parts by pixel wise analysis of camera images provided for triangulation measurement. ITG-Fachbericht. Bd. 281 Sensoren und Messsysteme, S. 203-206, 2018

[2] McCrackin F., Passaglia E., Stromberg S., Steinberg $\mathrm{H}$.: Measurement of the thickness and refractive index of very thin films and the optical properties of surfaces by ellipsometry. Journal of Research of the National Bureau of Standards Section A: Physics and Chemistry, 1963. doi: 10.6028/jres.067a.040

[3] Hlubina P., Luňáček J., Ciprian D., Chlebus R.: Spectral interferometry and reflectometry used to measure thin films. Applied Physics B, Bd. 92, Nr. 2, S. 203-207, 2008.

[4] Ghim Y., Kim S.-W.: Fast, precise, tomographic measurements of thin films. Applied Physics Letters, Bd. 91, Nr. 9, 2007. doi: 10.1063/1.2776015

[5] Quinten M.: Optische Schichtdickenbestimmung. Optik \& Photonik, Bd. 9, Nr. 1, S. 53-57, 2014. doi: 10.1002/opph.201300037

[6] Ghim Y., Kim S.-W.: Thin-film thickness profile and its refractive index measurements by dispersive white-light interferometry. Optics Express, Bd. 14, Nr. 24, 2006. doi: 10.1364/oe.14.011885

[7] Cardin J., Leduc D.: Determination of refractive index, thickness, and the optical losses of thin films from prism-film coupling measurements, Applied Optics, Bd. 47, S. 894, 2008. doi: 10.1364/ao.47.000894

[8] Metzner S., Hausotte T.: Evaluation of influences of thin lubricant on fringe projection measurements. Optics \& Photonics Yokohama, OPTM 2019, 2019.

[9] Jakob, G.: Koaxiale interferometrische Schichtdickenmessung. Photonik, Nr. 3, 2000

[10] Quinten, M.: Optische Schichtdickenmessung mit miniaturisierten Spektrometern. Books on Demand, 2015 S.32. ISBN: 978-3734783869 